\title{
Suspected ovarian cancer in a young patient with a congenital heart defect and a history of cardiac surgery and pacemaker implantation - difficult therapeutic decisions
}

\author{
Kamila Kaźmierczak, Tomasz Stempniewski, Joanna Kufel-Grabowska, \\ Błażej Nowakowski \\ Greater Poland Centre of Oncology in Poznan
}

Correspondence: Joanna Kufel-Grabowska Greater Poland Centre of Oncology in Poznań 61-866 Poznań, ul. Garbary 15 e-mail:joannakufel@googlemail.com

Received: 3.04.2018.

Accepted:

4.06.2018.

DOI: 10.24292/01.OR.040618 Copyright @ Medical Education. All rights reserved.

\section{ABSTRACT}

After uterine cancer, ovarian cancer is the second most common female reproductive tract malignancy. In Poland, in 2015, there were 3735 new cases and 2738 deaths from ovarian cancer. Over 80\% of all cases are diagnosed in women aged over 50 , whereas only $6 \%$ - before 35 years of age. Most cases are diagnosed, when clinical symptoms have already appeared, and the disease is at least locally advanced.

The case presented below describes a very young 28-year-old woman with a tumour in the pelvis, ascites and very high Ca 125 and HE 4 levels. The clinical symptoms as well as laboratory and imaging tests were suggestive of epithelial ovarian cancer that required urgent surgery due to progression of the disease.

The surgery posed a significant risk of complications, but owing to the deterioration of the patient's condition it was considered necessary. The patient was born with a severe, complex heart defect, had undergone two cardiac surgeries, and had a pacemaker (first VVIR, then DDD). She was also intensively treated for iatrogenic HCV infection and hypothyroidism.

A multidisciplinary team (a gynecologist, cardiologist and anesthetist) were behind the decision to carry out a major gynecological operation which ended with success. For the past 6 years, the patient has been under constant oncological follow-up and enjoys a good health.

Key words: ovarian cancer, congenital heart defect, Ca 125 marker, endometriosis 


\section{INTRODUCTION}

Ovarian cancer is one of the most common malignancies in postmenopausal women in Poland. It usually affects women over the age of 50, and rarely those under the age of 35 [1]. Each year, 3735 new cases are diagnosed in Poland, resulting in 2728 deaths annually [2]. The high mortality is primarily associated with the late diagnosis of the disease, which is oligosymptomatic at the initial stages, and the few symptoms that do occur in the process are non-specific. However, when abdominal pain sets in, abdominal circumference increases due to fluid accumulation, one is weakened and experiences reduced appetite, the risk of local or systemic advancement of a malignant disease is higher [1].

The main prognostic factors in ovarian cancer patients include: clinical advancement stage, histological type of the tumour, tumour grading, size of post-operative residual lesions, patient age, and BRCA1 or BRCA2 mutation status. The best prognosis is associated with an optimum surgical procedure, i.e. one without macroscopic neoplastic lesions left behind, followed by cytostatic treatment. In rare occasions, physicians may decide not to administer chemotherapy, in particular with respect to the G1 tumours limited to the ovaries [3].

In the case described below, the patient's general symptoms were suggestive at least of a local advancement of neoplastic disease, and her worsening condition required an urgent surgical intervention, which despite the patient's young age, was associated with a high risk of perioperative complications.

\section{CASE DESCRIPTION}

A 28-year-old female patient was urgently referred to the Department of Surgical, Oncological and Endoscopic Gynecology of the Greater Poland Cancer Center with a pelvic tumour diagnosis. The test performed revealed very high concentrations of the following markers: Ca 125 - $7826 \mathrm{U} / \mathrm{ml}$, and Ca 19-9 - $702 \mathrm{U} /$ $\mathrm{ml}$. The patient's general condition was average. She was weakened, and complained about a worsened exercise tolerance. The physical examination revealed an abdomen that was raised above the level of the thorax, tender, but without distinct peritoneal signs.

The gynecological examination performed upon admission revealed the following findings: vulva and perineum without pathological lesions, nodular lesions involving the posterior uterine ligaments, uterine body with limited mobility, and difficult to assess due to the tumour inside the pelvis.
The patient complained about irregular and painful menses, she had not yet given birth, and had had no miscarriages. Abdominal and pelvic ultrasound revealed a normal size and shape uterine body, with a $3.5 \mathrm{~cm}$ length, thin endometrium, and a normal cervix. Behind the uterus, there was a lesion indicative of endometriosis involving the uterosacral ligaments, and additionally an appendix with thickened walls and dilated lumen. There was a significant amount of free fluid of high viscosity within the peritoneum, possibly of mucous character. The small pelvis was filled with a cystic structure, ca. $8 \mathrm{~cm}$ in diameter, with multiple septa and densities corresponding with mucous masses. The above described lesion was not fully encapsulated. Within the fluid masses, a normal size right ovary was visualized. Additionally, there was thickened omentum of increased echogenicity, without focal lesions. The ultrasound scan did not reveal any other abnormalities pertaining to the other abdominal organs, and the retroperitoneal lymph nodes were not enlarged.

Chest X-ray revealed metallic sutures in the sternum, shadow of a cardiac pacemaker, and a low-enhanced and well-circumscribed oval shadow sized $34 \times 24 \mathrm{~mm}$ in the upper pole of the left pulmonary hilus, indicative of the presence of an enlarged lymph node or vascular shadow. There were no focal densities in the lungs. Costodiaphragmatic angles were visible shallower, the heart was not enlarged, and there was an elongated thoracic aorta. Both the clinical picture and the additional tests were suggestive of a malignant tumour of the left ovary.

The above clinical picture was also indicative of metastatic lesions within the peritoneum. Due to a reasonable suspicion of advanced neoplastic disease, surgical treatment was proposed to the patient, with a possibility of removing the uterus, both of the ovaries and oviducts, and all visible lesions from the peritoneal cavity.

Despite her young age, the patient had a significant cardiovascular history. She was born with a complex cardiac defect, i.e. double outlet right ventricle (DORV), pulmonary stenosis, and atrial septal defect (ASD II). When she was 15, she underwent a VVIR (ventricular inhibited pacing) pacemaker implantation due to a third-degree atrioventricular block. 2 years later, she had a pacemaker reimplanted due to staphylococcemia. When aged 25 , she underwent a yet another surgery involving battery replacement and a change of the pacing system to DDD pacemaker (pacing atria and ventricles).

When the patient was referred to the Greater Poland Cancer Center, she was also in the course of interferon a therapy for iatrogenic hepatitis $\mathrm{C}$ infection. 
Before the planned surgery, due to her history of prior cardiac interventions, the patient was referred for cardiovascular consultation, which included a general medical examination and an echocardiography scan. The echocardiogram revealed normal function of both ventricles and all valves. The patient's general condition was relatively good, and her functional status was assessed as NYHA II. Due to the presence of the pacemaker, it was suggested that electrocoagulation should be limited to the necessary minimum.

The patient was also consulted by an anesthesiologist, and her status was assessed based on the score established by the American College of Surgeons within the framework of the National Surgical Quality Improvement Program (ASC NSQIP). In the case of our patient, the overall risk of complications was significantly increased, amounting to $19.7 \%$, compared with the average risk of $9.9 \%$ for the planned procedure, whereas the risk of severe complications was twice as high as the population average (15.2\% vs. $7 \%)$ [4].

Despite the very high risk of complications, the physicians decided in favor of the surgery, which appeared to be the only therapeutic option available to the patient.

During the surgery, a significant amount of hemolyzed blood with clots was found in the peritoneal cavity, covering the surface of the greater omentum and intestinal loops, and numerous adhesions between the intestinal loops. Within the left ovary, there was a ruptured tumour in the form of an endometrial cyst connected with adhesions to the loops of the ileum and sigmoid. The external and internal surface of the cyst was smooth. The remaining abdominal organs showed no macroscopic lesions. The blood clots were evacuated, the intestinal loops were dissected free from the cyst, the cyst was excised together with the ovary, and handed over for intraoperative examination, whose result indicated a benign lesion.

The postoperative histopathology report confirmed the diagnosis of a left ovarian endometrial cyst with endometrial infiltration within the pelvis. There were no perioperative complications, and the wound healed well. 6 months afterwards, the levels of Ca 125 and Ca 19-9 went back to normal.

\section{DISCUSSION}

The above presented case of a young patient constituted a considerable diagnostic and therapeutic challenge. The symptoms that the patient presented as well as the additional tests per- formed were indicative of an advanced neoplastic process. An in-depth cardiac assessment was necessary as well as an analysis of the possible adverse events resulting from the use of interferon a in the course of hepatitis $C$ treatment.

The patient was born with a rare DORV heart defect, in which the aorta and the pulmonary artery both connect, entirely or primarily, to the right ventricle. Typical symptoms of the condition include: cyanosis, dyspnea, and reduced exercise tolerance, resulting in the baby's fatigability during feeding. The diagnosis is based on echocardiography, revealing both of the great arteries arising from the right ventricle. In complex cases, cardiac catheterization, $\mathrm{MRI}$ and/or $\mathrm{CT}$ is necessary. The treatment of choice is surgical management. Following surgical correction of the defect, the prognosis is good. A significant problem, following surgical treatment, may be life-threatening arrhythmia, requiring pacemaker implantation, which is exactly what happened in the case of our patient [5].

The above discussed cardiovascular comorbidities meant that the planned gynecological surgery was burdened with a significantly increased risk of perioperative complications, but it was successful, and the result of post-operative histopathology report was a surprise to the entire team, as it confirmed the presence of a benign ovarian lesion, and endometrial infiltrative lesions in the small pelvis.

Deep infiltrative endometriosis (DIE) is a severe form of endometriosis that infiltrates, inter alia, the uterosacral ligaments, bladder or rectum. The symptoms are non-specific, and often precede diagnosis by a few years, with one of the markers that may be indicative of the right diagnosis being the level of Ca 125 antigen, which is a mucin-type glycoprotein, tested mainly in patients with ovarian tumours. Presently, the antigen is believed to be the best biochemical marker for non-mucinous ovarian cancer. It is also elevated in inflammatory conditions of the small pelvis, endometriosis, uterine myoma, during menses, and in the first trimester of pregnancy. Additionally, elevated levels of the marker have been reported in a number of non-gynecologic conditions, including liver cirrhosis and cancer, pancreatic, lung and gastric cancers, lymphoma, and heart failure [6].

In the above described case, the level of Ca 125 significantly exceeded the values observed in patients with the diagnosis of DIE or heart failure, in whose case Ca 125 goes up to a few hundred $\mathrm{U} / \mathrm{ml}$, while in our patient it was as high as $7826 \mathrm{U} / \mathrm{ml}$, which clearly suggested coexistence of endometriosis and cancer, thus prompting the physicians to carry out a difficult surgery. In heart 
failure, elevated levels of Ca 125 often reflect an exacerbation of symptoms, and more serious disorders observed in echocardiogram tests. In the case of our patient, the echocardiogram test did not reveal any significant abnormalities, and the $\mathrm{N}$-terminal pro-brain natriuretic peptide (NT-proBNP) concentration was not measured. Its elevated level might have been indicative of heart failure, while normal values would point towards a non-cardiac cause behind the high Ca 125 levels [7]. Concurrence of internal and cardiovascular conditions, ovarian tumour and endometriosis resulted in a clinical picture that was suggestive of an advanced neoplastic process, with the suspicion additionally reinforced by the additional tests performed. The well-grounded decision to make an attempt at surgical management of the patient's condition, and success of the procedure, made it possible for the patient to resume her normal activities with a good performance status.

\section{CONCLUSIONS}

Treating cancer patients with a history of significant internal disease requires special attention and consideration on the part of the attending physicians, and the key to success is interdisciplinary cooperation. The above described patient is in a good physical and psychological condition, and remains under continuous care of her doctors.

\section{References}

1. Reid BM, Permuth JB, Sellers TA. Epidemiology of ovarian cancer: a review. Cancer Biol Med 2017; 14: 9-32.

2. Krajowy Rejestr Nowotworów [online: http://onkologia.org.pl/raporty/].

3. Crijns PG, Boezenb HM, Schouten JP et al. Prognostic factors in ovarian cancer: current evidence and future prospects. Eur J Cancer Supl 2003; 1(6): 127-145.

4. Khuri SF, Henderson WG, DePalma RG et al. Participants in the VA National Surgical Quality Improvement Program. Determinants of long-term survival after major surgery and the adverse effect of postoperative complications. Ann Surg 2005; 242: 326-241.

5. Grupa Robocza Europejskiego Towarzystwa Kardiologicznego (ESC) do spraw leczenia dorosłych pacjentów z wrodzonymi wadami serca: Wytyczne dotyczące leczenia dorosłych pacjentów z wrodzonymi wadami serca (nowa wersja - 2010). Kardiol Pol 2010; 68(supl IX): 639-696.

6. Oliveira MAP, Raymundo TS, Soares LC et al. How to Use CA-125 More Effectively in the Diagnosis of Deep Endometriosis. Biomed Res Int 2017; 2017: 9857196.

7. Zhuang J, Faggiano P, Li Q et al. Insights into the clinical implications of carbohydrate antigen 125 as a biomarker of heart failure: a meta-analysis and systematic review of published studies. J Cardiovasc Med 2014; 15: 864-872.

Authors' contributions: Kamila Kaźmierczak 30\%; Tomasz Stempniewski 30\%; Joanna Kufel-Grabowska 20\%;

Błażej Nowakowski 20\%. Conflict of interests: None.

Financial support: 